\title{
Reduced Object Related Negativity Response Indicates Impaired Auditory Scene Analysis in Adults with Autistic Spectrum Disorder
}

Auditory Scene Analysis provides a useful framework for understanding atypical auditory perception in autism. Specifically, a failure to segregate the incoming acoustic energy into distinct auditory objects might explain the aversive reaction autistic individuals have to certain auditory stimuli or environments. Previous research with non-autistic participants has demonstrated the presence of an Object Related Negativity (ORN) in the auditory event related potential that indexes pre-attentive processes associated with auditory scene analysis. Also evident is a later P400 component that is attention dependent and thought to be related to decision-making about auditory objects. We sought to determine whether there are differences between individuals with and without autism in the levels of processing indexed by these components. Electroencephalography (EEG) was used to measure brain responses from a group of 16 autistic adults, and 16 age- and verbal-IQ-matched typicallydeveloping adults. Auditory responses were elicited using lateralized dichotic pitch stimuli in which inter-aural timing differences create the illusory perception of a pitch that is spatially separated from a carrier noise stimulus. As in previous studies, control participants produced an ORN in response to the pitch stimuli. However, this component was significantly reduced in the participants with autism. In contrast, processing differences were not observed between the groups at the attention-dependent level (P400). These findings suggest that autistic individuals have difficulty at segregating auditory stimuli into distinct auditory objects, and that this difficulty arises at an early pre-attentive level of processing. 
1

Veema Lodhia ${ }^{\mathrm{a}}$, Jon Brock ${ }^{\mathrm{bc}}$, Blake W. Johnson ${ }^{\mathrm{bc}} \&$ Michael J. Hautus $^{\mathrm{a}^{*}}$

4

5

6

7

8

9

10 Running head: Auditory scene analysis in ASD

${ }^{\text {a }}$ Research Centre for Cognitive Neuroscience, School of Psychology, The University of Auckland, New Zealand.

${ }^{\mathrm{b}}$ ARC Centre of Excellence in Cognition and its Disorders,

${ }^{\mathrm{c}}$ Department of Cognitive Science, Macquarie University, Sydney, Australia

* Corresponding author:

12 Michael J. Hautus

13 School of Psychology (City),

14 The University of Auckland,

15 Private Bag 92019,

16 Auckland, 1142,

17 New Zealand.

$18 \quad$ Tel. +6493737599 ext. 85924

19 Fax. +64 93737450

20 Email: m.hautus@auckland.ac.nz 


\section{Introduction}

Autism is a developmental disorder that is defined and diagnosed in terms of impairments in social interaction and communication co-occurring with restricted behaviours and interests (American Psychiatric Association, 1994, 2013). In addition to these core diagnostic 'symptoms', many individuals with autism also experience hyper- or hypo-sensitivities in visual, auditory, and tactile domains (Talay-Ongan, \& Wood, 2000; Grandin, \& Scariano, 1986; Rosenhall, Nordin, Sandstrom, Ahlsen, \& Gillberg, 1999). Indeed, the recent revision of the DSM-5 (2013) diagnostic criteria for Autism Spectrum Disorder now makes explicit reference to sensory symptoms. Atypical auditory processing is particularly well documented. Many autistic individuals experience a distressing hyper-reactivity to noise (Grandin \& Scariano, 1986; Rosenhall et al., 1999) and several studies have reported that autistic individuals have difficulty extracting relevant auditory information (i.e., speech) in the presence of competing background noise (Boatman, Alidoost, Gordan, Lipsky, \& Zimmerman, 2001; Alcantara, Weisblatt, Moore, \& Bolton, 2004; Teder-Salejarvi, Pierce, Courchesne, \& Hillyard, 2005; Groen, van Orsouw, ter Huurne, Swinkels, van der Gaag, Buitelaar, \& Zwiers, 2009).

In the current study, we investigated auditory processing in autism within the context of Bregman's (1990) auditory scene analysis framework. According to Bregman, auditory perception involves grouping the incoming acoustic information into distinct auditory "objects" that correspond to inferred events in the listener's environment. This grouping occurs across time, space, and frequency and is determined by gestalt principles (such as similarity and temporospatial proximity) as well as attention and top-down effects of prior knowledge. Traditionally, auditory scene analysis has been investigated using behavioural methods in which participants report what they perceive as a function of stimulus manipulations. However, such methods are likely to be inappropriate for individuals with developmental disorders such as autism, who may be unable to provide an accurate introspective report of their perceptual experience. For this reason, investigations of auditory scene analysis in autism have measured auditory grouping indirectly via the measurement of brain responses.

In a 2005 study, Teder-Salejarvi et al. reported that, amongst individuals with autism, brain responses to sounds emanating from attended versus ignored spatial locations were indistinguishable. The authors concluded that the ability to focus auditory attention in complex 
acoustic environments is impaired in autism. However, this result could also indicate a problem with low-level perceptual segregation of the two sources. Subsequently, Lepistö et al. (2009) investigated auditory streaming using the mismatch negativity (MMN) paradigm. Adults with autism evidenced a typical MMN response to pitch deviants in a sequence of tones. However, this effect was eliminated when a separate stream of much higher tones was overlain, suggesting that the participants with autism did not segregate the sounds into separate auditory streams.

The current study investigated concurrent auditory segregation in adolescents and young adults with autism via the dichotic pitch paradigm. Dichotic pitch refers to the perception of pitches from stimuli that do not contain monaural cues to pitch (Bilsen, 1976; Cramer \& Huggins, 1958; Dougherty, Cynader, Bjornson, Edgell, \& Giaschi, 1998). Time-shifted dichotic pitch is created by presenting to each ear copies of broadband noises that have identical spectra but contain interaural time delays across a narrow frequency band. The frequency band containing the delay becomes perceptually separated from the remaining noise and is heard as a pitch with a tonal quality that is related to the centre frequency of the narrow frequency band (Johnson, Hautus, \& Clapp, 2003; Hautus \& Johnson, 2005). Because the time shift has no effect on the spectral content of the stimuli, any differential response can be assumed to reflect the cortical processes underlying auditory segregation (Hautus \& Johnson, 2005; Johnson et al., 2004; Hautus, Johnson, \& Colling, 2009).

Our previous research using such stimuli has demonstrated that perception of dichotic pitch is associated with a negative ERP component with a latency of about 150 - 250 ms (Hautus \& Johnson, 2005; Clapp, Johnson, \& Hautus, 2007; Johnson, Hautus, Duff, \& Clapp, 2007). This Object Related Negativity (ORN) was originally described by Alain, Arnott, \& Picton (2001) in the context of mistuned harmonics. It arises with or without attention to the auditory stimuli and is therefore assumed to represent a neurological marker of the pre-attentive stage of auditory scene segregation (Alain et al., 2001; Johnson \& Hautus, 2010).

A magnetic counter-part, the mORN, has also been found using magnetoencephalography (MEG) (Johnson \& Hautus, 2010; Johnson et al., 2004; Alain \& McDonald, 2007). In a recent MEG study, we found that children with autism failed to show an mORN to dichotic pitch stimuli, suggesting a failure of auditory segregation (Brock et al., 2013). However, results were 
inconclusive as the magnitude of the ORN was not significantly smaller than that evidenced by age-matched typically developing children.

The current study built on our earlier MEG study, using EEG to investigate the ORN. Rather than testing children, we tested young adults with autism, thereby allowing us to administer many more trials and achieve more reliable responses. Moreover, brain responses of adults are likely to be more consistent across individuals. Auditory evoked responses are typically mature by late adolescence (Mahajan \& McArthur, 2012) and studies comparing adolescents, young adults and middle-aged adults have found no evidence of developmental change in the ORN (Alain et al., 2001, 2003; Alain \& McDonald, 2007). To maximize the ORN response, we used "lateralized" dichotic pitch stimuli, whereby the broadband noise is also time-shifted in a direction opposite the narrow frequency band. In this case the segregation of pitch and noise is enhanced such that the listeners perceive the broadband noise lateralized to one side of auditory space and the pitch lateralized to the other side (see Figure 1; Johnson \& Hautus, 2010). This contrasts with the stimuli in our MEG study in which the pitch was lateralized but the residual noise was presented without an interaural timing difference and was therefore perceived as emanating from the centre of space.

As a final point of difference, we added a behavioural task in which participants were required to indicate via button press whether or not they heard the pitch sound. This contrasts with Brock et al. (2013) in which the participants were instructed to ignore the stimuli whilst watching a soundattenuated movie. This allowed us to directly compare behavioural and electrophysiological indices of auditory perception. Previous studies have indicated that the addition of a behavioural task elicits a positive component, termed the P400, with a latency of about $400-500 \mathrm{~ms}$ and, like the ORN, the P400 can be produced by ITD and inharmonicity. Unlike the ORN, the P400 is attention dependent, occurring only when participants are actively listening to (and discriminating between) stimuli. It is therefore thought to reflect the decision-making process related to the parsing of the incoming sound into concurrent perceptual objects (Alain et al., 2001; Hautus \& Johnson, 2005). 
109

110

111

112

113

114

115

116

117

118

119

120

121

122

Participants were 16 individuals with an Autism Spectrum Disorder (ASD) and 16 typicallydeveloping (TD) individuals. A further 5 participants with ASD were excluded because they were unable to satisfactorily discriminate dichotic pitch during the practice phase (see details below).

The two groups of 16 were matched on gender, age ( \pm 2 years), and handedness, determined by the Edinburgh Handedness Inventory (Oldfield, 1971).

Participants in the ASD group were recruited via adverts posted at Autism NZ, Altogether Autism, Autism House, Centre for Brain Research, and The University of Auckland. Participants gave their informed written consent, and all procedures were approved by The University of Auckland Human Participants Ethics Committee (Ref: 2009/537).

Exclusion criteria for the ASD participants included a co-morbid Axis 1 disorder and relevant Axis 3 diagnosis, hearing deficits and pharmacological treatment. For participants in the TD group, the exclusion criteria included personal or family history of neurological or psychiatric disorders, hearing deficits, and pharmacological treatment. Further inclusion criteria for both TD and ASD groups were (1) normal auditory acuity - hearing thresholds $\leq 25 \mathrm{~dB} \mathrm{HL}$, as assessed by an audiogram (Amplitude T-Series, Otovation, LLC, USA) for the standard range of $250-$ $8000 \mathrm{~Hz}$; (2) a full-scale mental ability score whose lower confidence bound was $\geq 80$; and (3) passing a pre-screening assessment demonstrating an ability to detect dichotic pitch.

All participants in the ASD group had been given a clinical diagnosis of autistic disorder $(N=3)$ or Asperger's disorder $(N=13)$ according to DSM-IV. Diagnoses were made by a clinical psychologist or paediatrician. As a further check, we determined that all participants met the cutoff for ASD on the Social Communication Questionnaire (SCQ - Lifetime scale $\geq 15$ ), which was completed by a parent or guardian at the first study meeting. The SCQ is based on the Autism Diagnostic Interview-Revised, with which it has good agreement (Bishop \& Norbury, 2002) ${ }^{1}$.

Table 1 summarizes the demographic and behavioural test results for both groups. No group differences were found for verbal or combined IQ as measured using the Wechsler Abbreviated Scales of Intelligence (Wechsler, 1999). A group difference was found for performance IQ, nevertheless the ASD group performed above average for their age group. 
Stimuli

140 Two independent broadband Gaussian noise bursts, each $500 \mathrm{~ms}$ in duration, were constructed at a sampling rate of $44.1 \mathrm{kHz}$, using LabVIEW software (National Instruments, Austin, Texas, USA). One noise burst was bandpass filtered with a centre frequency of $600 \mathrm{~Hz}$ and a bandwidth of $20 \mathrm{~Hz}$ using a fourth-order Butterworth filter. The other noise burst was notch filtered using the same filter characteristics. A copy was made of both noises (bandpass and notch), one copy of each type for each ear. For the target stimulus (noise plus pitch; two auditory objects) opposing temporal delays $( \pm 500 \mu \mathrm{s})$ were applied to the bandpass- and notch-filtered noises so that the resulting combination would create a noise lateralized to one side of auditory space and a pitch to the other side of auditory space. For control stimuli (noise alone; one auditory object) both the bandpass- and the notch-filtered noise were temporally delayed $(500 \mu \mathrm{s})$ to the same ear (Figure 1), resulting in noise lateralized to one side of space. The notch- and bandpass-filtered noise processes within each auditory channel were recombined, producing two spectrally flat noise processes, which were again bandpass filtered (fourth-order Butterworth filter) with a centre frequency of $600 \mathrm{~Hz}$ and bandwidth of $400 \mathrm{~Hz}$. The stimuli were windowed with a $\cos ^{2}$ function with $4 \mathrm{~ms}$ rise and fall times. The auditory stimuli were generated on two-channels of a 16-bit converter (Model DAQPad 6052E, National Instruments, Austin, TX). Programmable attenuators (Model PA4, Tucker-Davis Technologies, Alachua, FL) set the binaural stimuli to yield $70 \mathrm{~dB}$ SPL from insert earphones at the ear. (ER2, Etymotic Research Inc., Elk Grove Village, Illinois, USA).

\section{Behavioural task}

On each trial, participants indicated on a button box whether the stimulus presented consisted of one or two auditory objects. In an initial practice session, prior to EEG recording, participants completed four 100-trial blocks with feedback received after each trial. Five of the original 21 participants with ASD did not reach the criterion of 69 percent correct (approximately $d^{\prime}=1$; cf. Macmillan \& Creelman, 2005, p. 9) in the practice session and were therefore excluded from the 
168

169

170

171

172

173

174

175

176

EEG part of the study because they could not sufficiently discriminate between the two types of stimuli.

During the EEG recording, the task was similar, except that no feedback was given and the trial timed out after $1500 \mathrm{~ms}$ if no response was made. The inter-stimulus intervals were drawn from a rectangular distribution between $2000 \mathrm{~ms}$ and $3400 \mathrm{~ms}$. Participants completed four blocks of 256 trials, each of which took approximately 13 minutes to complete. Short breaks were given after each block.

\section{Electroencephalography}

EEG recordings were conducted in an electrically shielded room (Belling Lee - Model L3000, Enfield, England) using 128-chanel Ag/AgCl electrode nets (Tucker, 1993; Electrical Geodesics Inc., Eugene, Oregon, USA). EEG was recorded continuously (250-Hz sample rate; 0.1-100 Hz analogue bandpass) with Electrical Geodesics Inc. amplifiers (200-M $\Omega$ input impedance). Electrode impedances were kept below $40 \mathrm{k} \Omega$, an acceptable level for this system (Tucker, 1993). Common vertex $(\mathrm{Cz})$ was used as a reference. During the EEG, participants were asked to fixate on a cross, presented on a computer screen.

\section{Data analysis}

EEG files were segmented into $750 \mathrm{~ms}$ epochs (including a $100 \mathrm{~ms}$ pre-stimulus baseline) during which all ocular artifacts were corrected (Jervis, Nichols, Allen, Hudson, \& Johnson, 1985). Trials with channels marked as bad were dropped from the averaging process. $98 \%$ of trials remained for analysis from each group. Given that the ORN is elicited regardless of whether a task is performed, all trials were included, irrespective of response accuracy. ERPs were rereferenced to the average reference. ERPs from individual participants were combined to produce grand-averaged ERPs for each condition. Grand averaged data were then digitally filtered with a zero-phase-shift 3-pole Butterworth filter $(0.1$ - $30 \mathrm{~Hz}$; Alarcon, Guy, \& Binnie, 2000) and then re-referenced to the mean.

For statistical analysis, the electrode clusters of interest for the ORN and the P400 components were selected by combining all 32 participants' data for the No Pitch and the Pitch conditions. These grand averaged waveform topographic maps were then used to select a symmetrical cluster of electrodes that showed the greatest difference in mean amplitude between the No pitch and the 
Pitch conditions (left hemisphere electrodes: 7, 12, 13, 20, 28, 29, 30, 31, 37; right hemisphere: 5, $80,87,105,106,111,112,117,118)$. For each participant we then averaged across these channels to calculate a Pitch and a No-Pitch waveform. Time windows for the ORN and P400 components were determined based on the full width half max of the difference waveform for the combined group $(\mathrm{N}=32)$. For each participant, the magnitude of the two components was calculated as the area under the curve in the difference waveform.

As Kilner (2013) has recently pointed out, selecting channels and time windows based on the observed peaks inflates the likelihood of false-positives in the within-subjects effect (i.e., it increases the likelihood of finding a main effect of Condition when none exists). However, our aim was not to replicate the numerous previous studies demonstrating the existence of the ORN and P400 but rather to determine whether the components differed in magnitude across groups. Because our choices were all made based on the data averaged across both groups (and because the groups were of equal size), they should not increase the likelihood of a false positive group difference.

\section{Results}

\section{Behavioural performance}

ANOVA revealed a main effect of Group, $\left(\mathrm{F}(1,30)=12.75, \mathrm{p}<.001, \mathrm{~h}_{\mathrm{p}}{ }^{2}=.298\right)$, indicating that the TD group obtained a higher percentage correct score $(87.38 \%)$ than the ASD group $(70.38$ $\%)$.

\section{Event-related potentials}

Figure 2 shows the ERP waveforms for Typically Developing and ASD participants in response to Pitch and No Pitch (Control) stimuli. Typically developing participants showed an increased negativity (ORN) to the Pitch stimuli, coincident with the P2 and N2 peaks. This was followed by an increased positivity P400 at around $400 \mathrm{~ms}$. Waveforms for participants with ASD were similar overall, but there was little evidence of a differential response to Pitch and No Pitch stimuli. 
For the ORN time window, ANOVA confirmed a more negative response to Pitch compared with the No Pitch stimuli $\left(F(1,30)=34.87, p<.001, \mathrm{~h}_{\mathrm{p}}{ }^{2}=.538\right)$. There was no main effect of Group $\left(F(1,30)=0.79, p=.382, \mathrm{~h}_{\mathrm{p}}{ }^{2}=.026\right)$. However, as predicted, there was a significant Pitch $\times$ Group interaction $\left(F(1,30)=8.66, p=.006, \mathrm{~h}_{\mathrm{p}}{ }^{2}=.224\right)$, with a considerably larger effect of Pitch in the TD group (see Figure 3). Follow-up $t$-tests (two-tailed) indicated that the TD group showed a significant ORN $(t(15)=-6.43, p<.001)$ but the ASD group did not $(t(15)=-2.04, p=.059)$.

Figure 3 also indicates the presence of an outlier in the TD group, with an ORN $(-0.72 \mu \mathrm{V})$ that was considerably larger than that of the other participants. We therefore repeated the analyses with the outlier excluded. Critically, the Pitch x Group interaction remained significant $(F(1,29)$ $\left.=7.31, p=.011, \mathrm{~h}_{\mathrm{p}}{ }^{2}=.201\right)$ indicating that the group differences were not driven solely by this outlier.

Pearson's correlation analyses revealed that, within the ASD group, better behavioural performance during the EEG recording was associated with a more negative ORN $(r(16)=-.567$, $p=.022$ ). In other words, individuals with ASD who performed well on the task tended to show a typical ORN, whereas those who performed poorly demonstrated a reduced ORN (Figure 3). Within the TD group, the correlation was in the same direction but fell well short of significance $(r(16)=-.314, p=.237)$, perhaps reflecting ceiling effects on performance.

Further correlation analyses (see Table 2) showed no association between ORN magnitude and either age, scores on the Social Communication Questionnaire, verbal IQ, or performance IQ within the ASD group (minimum $p=.34$ ). Similar analyses of the TD group revealed a significant correlation between the ORN and verbal IQ $(r(16)=.691, p=.003)$ but this became non-significant when the outlier was excluded $(r(15)=.500, p=.057)$. All other correlations were non-significant, with or without the outlier.

Results for the P400 component were less clear-cut. ANOVA confirmed a more positive response to Pitch compared with the No Pitch stimuli $\left(F(1,30)=5.02, p=.033, \mathrm{~h}_{\mathrm{p}}{ }^{2}=.143\right)$. There was again no main effect of Group $\left(F(1,30)=0.01, p=.981, \mathrm{~h}_{\mathrm{p}}{ }^{2}=.000\right)$ but, unlike for the ORN, there was no Pitch $\times$ Group interaction $\left(F(1,30)=0.21, p=.650, \mathrm{~h}_{\mathrm{p}}{ }^{2}=.007\right)$. Follow-up t-tests 
253 (two-tailed) indicated that neither the TD group $(t(15)=1.79, p=.094)$ nor the ASD group $(t(15)$

$254=1.36, p=.195$ ) showed a significant effect of Pitch when considered in isolation. Correlations

255 between the P400 and measures of behavioural performance were not significant in either group,

256 although there was a marginally significant association with verbal IQ $(r(16)=.496, p=.051)$.

257 Given the large number of correlations performed, it would be unwise to draw any conclusions

258 based on this finding.

259

260

261

\section{Discussion}

Auditory Scene Analysis provides a useful framework for understanding atypical auditory perception in autism. Specifically, a failure to segregate the confusion of incoming auditory energy into distinct auditory objects might explain the aversive reaction autistic individuals have to certain auditory stimuli or environments. Our prediction in this study was that autistic individuals would evidence a reduced ORN, indicating a failure to segregate the dichotic pitch stimuli into spatially separate auditory objects. This proved to be the case. Where TD participants showed a significant ORN, the effect was reduced in adults with ASD, who did not themselves show a significant ORN.

As in previous studies, we focused on electrophysiological measures of auditory perception in order to avoid potential confounds such as task understanding and attention that might affect performance on behavioural tasks. However, there was, in fact, substantial agreement between behavioural and electrophysiological measures both at the group and the individual level. This indicates that, in the high-functioning adults tested here, the behavioural performance is a good indicator of underlying perceptual processes, and that together the two measures provide converging evidence for atypical perception, at least in a subgroup of individuals with ASD.

These results are also broadly consistent with our previous study in which we failed to find a significant ORN in a group of autistic children (Brock et al., 2013). The current results are, however, more compelling insofar as they revealed a significant group by condition interaction, which was only a trend in the earlier study ${ }^{2}$. It is not clear which of the various methodological differences might explain this difference in outcome. The current study used EEG rather than MEG, used lateralized noise rather than centralized noise, included more participants and more trials, and involved adults rather than children. Any or all of these differences could be relevant. 
Alternatively, given the variation in both the ORN and behavioural performance within our ASD group, as well as the inherent heterogeneity in the wider ASD population, results could simply reflect differences in sampling across the two studies. Also of potential significance is the absence of "gold standard" tests for diagnosing adults with autism, and that diagnoses of autism were made by several qualified professionals.

It is also difficult to be sure at this stage to what extent these findings are specific to the dichotic pitch paradigm or reflect auditory segregation more generally. Our ongoing research looks to address this issue by using other auditory stimuli that also produce an ORN. That being said, participants with ASD were all significantly above chance in the practice session, indicating that they were at least able to detect the inter-aural timing differences that gave rise to the dichotic pitch perception. The reduced ORN in their response suggests that, even though they were able to detect some difference between the pitch and control stimuli, their auditory systems did not fully segregate these two sound qualities (noise and pitch) into separate auditory objects. Rather, they are more likely to perceive a single auditory object that has both noise- and pitch-like qualities. The distinction in the qualities of this single object allows the behavioural task to be completed successfully; albeit with lower performance than the TD participants.

This interpretation would also be consistent with the absence of group differences in the later P400 component, which is thought to index the task-based decision. However, caution is required in interpreting the $\mathrm{P} 400$ responses given that neither group evidenced a significant $\mathrm{P} 400$ effect on their own, and that the P400 response did not correlate significantly with behavioural performance. Thus, it may simply be the case that the P400 response is unreliable, or that its latency or spatial distribution varies across individuals, meaning that we were unable to extract a measure of the P400 size that actually reflected the strength of the underlying neurophysiological processes

Our working hypothesis, therefore, is that ASD individuals have (or are more likely to have) difficulties in the segregation of auditory stimuli into distinct auditory objects. This ability is known to begin in infancy (Folland, Bulter, Smith, \& Trainor, 2012; Dermany, 1982; McAdams $\&$ Bertoncini, 1997) and continues to improve in conjunction with growth of neuronal connectivity in adolescence (Smith \& Trainor, 2011). Reduced ability to filter out and process multiple sounds may, therefore, be attributed to atypical brain development and growth in ASD. 
312 Source modelling suggests that the neural generators of the ORN are located in the posterior 313 supratemporal plane for dichotic pitch stimuli (Hautus \& Johnson, 2005), consistent with the 314 view that the planum temporale neural network has a functional role in concurrent sound 315 segregation (Alain et al., 2001; Griffiths \& Warren, 2002). Of note, there have been several 316 reports that individuals with ASD have a smaller planum temporale compared to typically 317 developing individuals (Rojas, Bawn, Benkers, Reite, \& Rogers, 2002; Rojas, Camou, Reite, \& 318 Rogers, 2005) although, without MRIs for the current participants, this remains speculative.

319 Further research is therefore required to determine how common the deficits in auditory object 320 processing are within the ASD population, and whether they relate at the individual level to atypical perceptual experiences. In particular, our study specifically concentrated on high functioning adults. It is unclear whether we would find similar pre-attentive processing difficulties with other ASD profiles such as younger children and lower functioning individuals. Some sub-groups within the autistic spectrum may have very different auditory perceptual experiences to those tested here.

It also remains to be established how specific these difficulties are to ASD. In a recent study, we found no difference in the ORNs generated by typically developing children and those with specific reading difficulties (Johnson et al., 2013). There are, however, many other conditions associated with atypical auditory processing, and affected individuals might show effects similar to those with autism (e.g., Elsabbagh, Cohen, \& Karmiloff-Smith, 2010; Goll, Crutch, \& Warren. 2010). These caveats notwithstanding, the current study adds to the growing body of evidence that atypical auditory perception associated with autism may be understood in terms of differences in auditory scene analysis. 


\section{Acknowledgements}

335 The authors gratefully acknowledge the assistance and support from Autism NZ, Altogether

336 Autism, Autism House, the participants and their families for supporting this research, and Ms

337 Chaturangi Nelumdeniya for assistance with the collection of data.

\section{$338 \quad$ Funding}

339 The work of BWJ and JB was supported by the Australian Research Council (ARC) Centre of

340 Excellence for Cognition and its Disorders (CE110001021) http://www.ccd.edu.au. JB was

341 supported by an ARC Australian Research Fellowship (DP098466). 


\section{References}

Alain C, Arnott SR, Picton TW. 2001. Bottom-up and top-down influenced on the auditory scene analysis: evidence from event related brain potentials. Journal of Experimental Psychology 27(5):1072 -1089.

Alain C, McDonald K. 2007. Age-related differences in neuromagnetic brain activity underlying concurrent sound perception. The Journal of Neuroscience 27:1308-1314.

Alain C, Theunissen EL, Chevalier H, Batty M, Taylor M. 2003. Developmental changes in distinguishing concurrent auditory objects. Cognitive Brain Research 16:210-218.

Alarcon G, Guy CN, Binnie CD. 2000. A simple algorithm for a digital three-pole Butterworth filter of arbitrary cut-off frequency: application to digital electroencephalography. Journal of Neuroscience Methods 104(1):35-44.

Alcantara JI, Weisblatt EJL, Moore BCJ, Bolton PF. 2004. Speech in noise perception in high functioning individuals with autism or Asperger's syndrome. Journal of Child Psychology and Psychiatry 45(6):1107-1114.

American Psychiatric Association. 1994. Diagnostic and Statistical Manual of Mental Disorders, Fourth Edition (DSM-IV). Washington, DC: American Psychiatric Publishing.

American Psychiatric Association. 2013. Diagnostic and Statistical Manual of Mental Disorders, Fifth Edition (DSM-5). Arlington VA: American Psychiatric Publishing.

Bastiaansen JA, Meffert H, Hein S, Huizinga P, Ketelaars C, Pijnenborg M, Bartels A, Minderaa R, Keysers C, de Bildt A. 2011. Diagnosing autism spectrum disorders in adults: the use of Autism Diagnostic Observation Schedule (ADOS) Module 4. Journal of Autism and Developmental Disorders, 41: 1256-1266.

Bishop DV, Norbury CF. 2002. Exploring the borderlands of autistic disorder and specific language impairment: A study using standardised diagnostic instruments. Journal of Child Psychology and Psychiatry 43:917-929.

Bilsen FA. 1976. Pronounced binaural pitch phenomenon. Journal of the Acoustical Society of America 59:467-468.

Boatman D, Alidoost M, Gordan B, Lipsky F, Zimmerman W. 2001. Enhanced pitch sensitivity in individuals with autism: A signal detection analysis. Journal of Cognitive Neuroscience $15: 226-235$.

Bregman AS. 1990. Auditory scene analysis. The perceptual organization of sound. Cambridge, Massachusetts: MIT Press.

Brock J, Bzishvili S, Reid M, Hautus M, Johnson BW. 2013. Atypical neuromagnetic responses to illusory auditory pitch in children with autism spectrum disorders. Journal of Autism and Developmental Disorders, 47(11): 2726-2731. 
Cramer EM, Huggins WH. 1958. Creation of pitch through binaural interaction. Journal of the Acoustical Society of America 30(5):413-417.

Clapp WC, Johnson BW, Hautus MJ. 2007. Graded cue information in dichotic pitch: effects on event-related potentials. NeuroReport 18(4):365-638.

Dougherty R, Cynader MS, Bjornson BH, Edgell D, Giaschi DE. 1998. Dichotic pitch: a new stimulus distinguishes normal and dyslexic auditory function. NeuroReport 9(13):30013005 .

Dermany L. 1982. Auditory stream segregation in infancy. Infant Behaviour \& Development 48:261-276.

Elsabbagh M, Cohen H, Karmiloff-Smith A. 2010. Discovering structure in auditory input: evidence from Williams Syndrome. American Journal on Intellectual and Developmental Disabilities 115(2): 128-139.

Folland N, Bulter BE, Smith NA, Trainor LJ. 2012. Processing simultaneous auditory objects in infancy: Music and mistuned harmonics. Journal of the Acoustical Society of America 313:993-997.

Goll JC, Crutch SJ, Warren JD. 2010. Central auditory disorders: toward a neuropsychology of auditory objects. Current Opinion in Neurology 23(6): 617-627.

Grandin T, Scariano MM., 1986. Emergence: Labelled autistic. New York: Arena Press.

Griffiths TD, Warren JD. 2002. The planum temporale as a computational hub. Trends in Neurosciences 25:348-353

Groen WB, van Orsouw L, ter Huurne N, Swinkels S, van der Gaag RJ, Buitelaar JK, Zwiers MP. 2009. Intact spectral but abnormal temporal processing of auditory stimuli in autism. Journal of Autism and Developmental Disorders 39(3):742-750.

Hautus MJ, Johnson BW, Colling LJ. 2009. Event-related potentials for interaural timing differences and spectral cues. NeuroReport 20:951-956.

Hautus MJ, Johnson BW. 2005. Object-related brain potentials associated with the perceptual segregation of dichotically embedded pitch. Journal of the Acoustical Society of America 117(1):275-280.

Jervis BW, Nichols MR, Allen EM, Hudson NR, Johnson TE. 1985. The assessment of two methods for removing eye movement artifacts from the EEG. Electroencephalography and Clinical Neurophysiology 61:444-452.

Johnson BW, Hautus MJ. 2010. Processing of binaural spatial information in human auditory cortex: neuromagnetic responses to interaural timing and level difference. Neuropsychologia 48:2610-2619. 
411

412

413

414

415

416

417

418

419

420

421

422

423

424

425

426

427

428

429

430

431

432

433

Johnson BW, Hautus MJ, Clapp WC. 2003. Neural activity associated with binaural processes for the perceptual segregation of pitch. Clinical Neurophysiology 114:2245-2250.

Johnson BW, Hautus MJ, Duff DJ, Clapp WC. 2007. Sequential processing of interaural timing differences for sound source segregation and spatial localization: evidence from eventrelated cortical potentials. Psychophysiology 44:541-551.

Johnson BW, McArthur G, Hautus M, Reid M, Brock J, Castles A, Crain S. 2013. Lateralized auditory brain function in children with normal reading ability and in children with dyslexia. Neuropsychologia 51(4):633-641.

Johnson BW, Muthukumaraswamy SD, Hautus MJ, Gaetz WC, Cheyne DO. 2004. Neuroimaging responses associated with perceptual segregation of pitch. Neurology and Clinical Neurophysiology 33:1-4.

Kilner, JM. 2013. Bias in a common EEG and MEG statistical analysis and how to avoid it. Clinical Neurophysiology 124(10):2062-2063.

Lepistö T, Kuitunen A, Sussman E, Saalasti S, Jansson-Verkasalo E, Nieminen-von Wendt T, Kujala T. 2009. Auditory stream segregation in children with aspergers syndrome. Biological Psychology 82:301-307.

Lord C, Rutter M, DiLavore PC, Risi S. 1999. Autism diagnostic observation schedule. Manual. Los Angeles, CA: Western Psychological Services.

Macmillan NA, Creelman CD. 2005. Detection Theory: A User's Guide (2nd edn.). Mahwah, New Jersey, USA: Lawrence Erlbaum Associates.

Mahajan Y, McArthur G. 2012. Maturation of auditory event-related potentials across adolescence. Hearing Research 294:82-94.

McAdams S, Bertoncini J. 1997. Organization and discrimination of repeating sound sequences by newborn infants. Journal of the Acoustical Society of America 102:2945-2953.

Oldfield RC. 1971. The assessment and analysis of handedness: The Edinburgh inventory. Neuropsychologia 9:97-113.

Rojas DC, Bawn SD, Benkers TL, Reite ML, Rogers SL. 2002. Smaller left hemisphere planum temporale in adults with autistic disorder. Neuroscience Letters 328:237-240.

Rojas DC, Camou SL, Reite ML, Rogers SL. 2005. Planum temporale volume in children and adolescents with autism. Journal of Autism and Developmental Disorders 35(4):479-486.

Rosenhall U, Nordin V, Sandstrom M, Ahlsen G, Gillberg C. 1999. Autism and hearing loss. Journal of Autism and Developmental Disorders 29:349-357.

Smith NA, Trainor LJ. 2011. Auditory stream segregation improves infants selective attention to target tones and distracters. Infancy 16:1-14. 
445 Talay-Ongan A, Wood K. 2000. Unusual sensory sensitivities in autism: a possible crossroads. International Journal of Disability, Development and Education 47(2):201-212.

447 Teder-Salejarvi WA, Pierce KL, Courchesne E, Hillyard SA. 2005. Auditory spatial localization 448 and attention deficits in autistic adults. Cognitive Brain Research 23:221-234.

449 Tucker DM. 1993. Spatial sampling of head electrical fields: the geodesic sensor net. $450 \quad$ Electroencephalography \& Clinical Neurophysiology 87(3):154-163.

451 Wechsler D. 1999. Wechsler abbreviated scale of intelligence (WASI). New York: The $452 \quad$ Psychological Corporation. 


\section{Footnotes}

454 1. A limitation of the current study (and indeed all other studies of adults with autism) is the lack 455 of an "objective" verification of autism diagnosis. The Autism Diagnostic Observation Schedule 456 (Lord et al., 1999) is considered by some researchers to be the gold standard for autism diagnosis. 457 However, studies suggest that in adults it fails to discriminate between autism and other 458 conditions such as schizophrenia that have overlapping symptoms (Bastiaansen et al., 2011).

459 2. In our previous MEG study, analyses were conducted by a bootstrapping analysis of the 460 difference waveforms. To allow a more direct comparison with the current study, we re-analysed 461 the MEG data, calculating the mean amplitude of the source waveform for the right hemisphere 462 between 250 and 360 milliseconds. This choice was based on analysis of data from a larger 463 sample of typically developing children (Johnson et al., 2013) which showed a significant ORN 464 in this window. Consistent with the current study, we found no ORN in the ASD group $(t(9)=$ $4650.23, p=.827)$, but the group by condition interaction was also non-significant $(F(1,18)=1.48$, $\left.466 p=.239, \square_{\mathrm{p}}^{2}=.076\right)$. 


\section{Table 1 (on next page)}

Demographic and cognitive characteristics of the TD and ASD groups. 


\begin{tabular}{|c|c|c|c|c|c|c|c|}
\hline \multirow{2}{*}{ Measure } & \multirow{2}{*}{$\begin{array}{c}\operatorname{ASD}(N=16) \\
M(S D)\end{array}$} & \multirow{2}{*}{$\begin{array}{c}\text { TD }(N=16) \\
M(S D)\end{array}$} & \multicolumn{2}{|c|}{ Range } & \multicolumn{3}{|c|}{ Independent $t$-test } \\
\hline & & & Min & Max & $t$ & $d f$ & $p$ \\
\hline Age (years) & $22.19(5.99)$ & $22.69(5.20)$ & 16 & 34 & 0.59 & 30 & .80 \\
\hline $\begin{array}{l}\text { Handedness } \\
100 \%=\text { right }\end{array}$ & $75.69(54.70)$ & $68.62(62.77)$ & -100 & 100 & -0.34 & 30 & .74 \\
\hline Verbal IQ & $119.50(18.69)$ & $118.38(14.89)$ & 84 & 140 & -0.19 & 30 & .85 \\
\hline Performance IQ & $107.25(13.76)$ & $116.25(9.95)$ & 72 & 131 & 2.12 & 30 & .04 \\
\hline Combined IQ & $114.75(16.64)$ & $120.31(12.27)$ & 79 & 137 & 1.08 & 30 & .29 \\
\hline SCQ & $23.06(5.22)$ & - & 15 & 33 & - & - & - \\
\hline
\end{tabular}




\section{Table 2 (on next page)}

Correlations for each group between electrophysiological measures (ORN and P400) and participant demographics and accuracy. 


\begin{tabular}{ccccc}
\hline Measure & ASD & TD & ASD & P400 \\
\hline Accuracy & $-.567^{*}$ & -.314 & .222 & TD \\
\hline Age & .253 & .046 & -.341 & .187 \\
\hline Verbal IQ & .073 & $.691^{* *}$ & .496 & .295 \\
\hline Performance IQ & -.049 & .226 & .393 & .335 \\
\hline SCQ & -.148 & N/A & -.148 & N/A \\
\hline
\end{tabular}




\section{Figure 1}

Schematic representations of the dichotic pitch stimuli.

These representations indicate the nature of the percept associated with the four stimulus configurations. The top panels show the No Pitch (or control) stimuli that lead to the perception of a noise lateralized to one side of auditory space. The bottom panels show the Pitch stimuli which also lead to the perception of a noise, but in addition, a pitch is perceived lateralized to the side opposite the noise. (Noise represented by \#\#\# and Pitch represented by §).

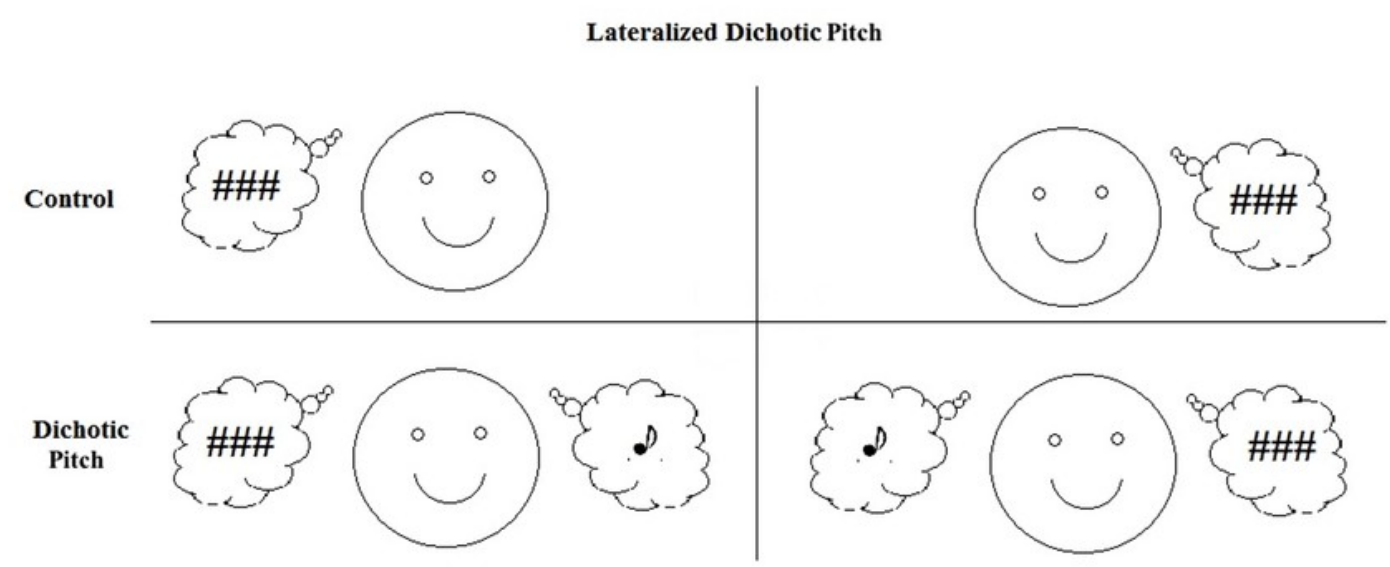




\section{Figure 2}

Event related potential waveform graphs.

Grand averaged ERP (-100-750 ms) graphs of the No Pitch and Pitch stimuli for the TD group and ASD group. Shaded regions indicate the time windows used for calculating the ORN (168-284 ms) and P400 (404-520 ms).

TD Group

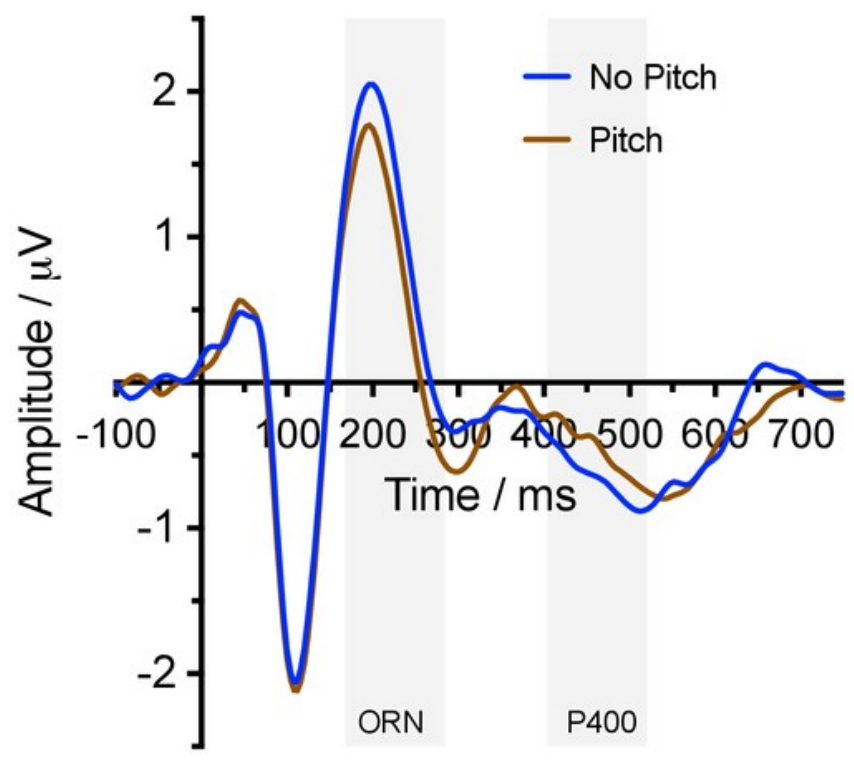

ASD Group

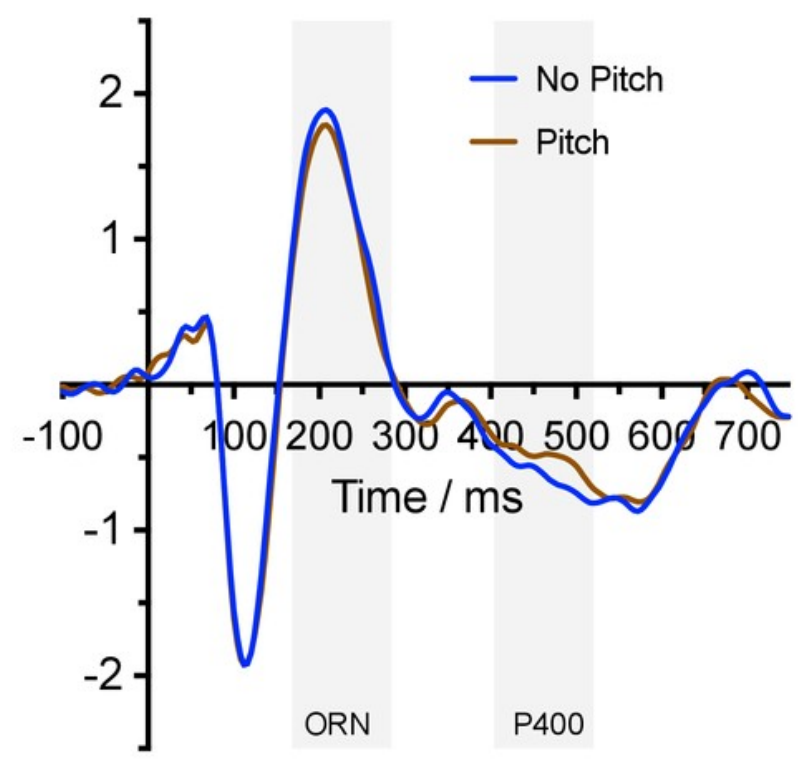




\section{Figure 3}

Association between behavioural performance during the EEG recording and the magnitude of the ORN.

Regression line (and confidence intervals) is fitted to the ASD data (circles). Boxplots show the distributions of behavioural performance (right panel) and ORN (top panel).

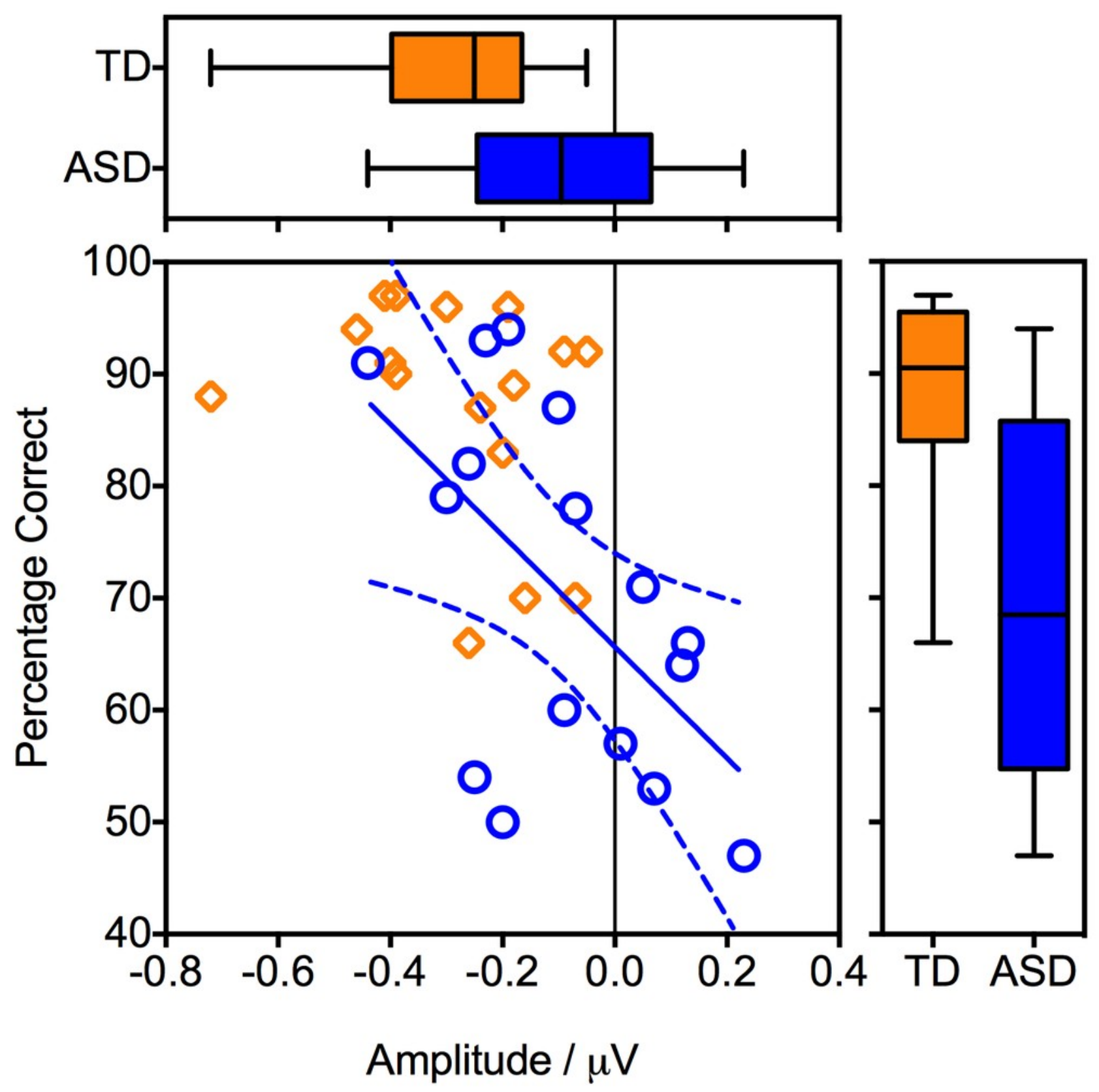

